\title{
SURVEY OF SHORT-HORNED GRASSHOPPERS (ACRIDIDAE) FROM DAKSHINA KANNADA DISTRICT, KARNATAKA
}

\author{
Seetharama Mayya ${ }^{1}$, K.S. Sreepada ${ }^{2}$ and M. Jayarama Hegde ${ }^{3}$ \\ ${ }^{1}$ Professor \& Head, Department of Zoology, SVS College, Bantwal, Dakshina Kannada, Karnataka 574211, India \\ ${ }^{2}$ Department of Applied Zoology, Mangalore University, Mangalagangotri, Karnataka 574199, India \\ ${ }^{3}$ Department of Bio-technology, P.A. College of Engineering, Nadupadavu, Mangalore, Karnataka, India \\ Email: ${ }^{1}$ smayya_svscollege@yahoo.co.in
}

\begin{abstract}
Twenty-eight species of short-horned grasshoppers (Acrididae) were recorded from $250 \mathrm{~km}^{2}$ area of Dakshina Kannada district, Karnataka, from September 2000 to June 2002. The surveys in 12 localities indicated differences in diversity and density of grasshoppers pointing to areas with anthropogenic influence, pollution and agriculture to be less diverse compared to less disturbed and polluted areas.
\end{abstract}

\section{KEYWORDS}

Acrididae, catalogue, Dakshina Kannada, diversity, Karnataka, Mangalore, Short-horned grasshopper, survey,

Dakshina Kannada a coastal district of Karnataka in southern India experiences an average annual rainfall of $4400 \mathrm{~mm}$. The temperature ranges from $26.1^{\circ} \mathrm{C}$ to $36^{\circ} \mathrm{C}$ and humidity ranges from $70-85 \%$. The important agricultural and horticultural plants include paddy, coconut, arecanut, cashew and mango among others. The district also hosts many species of grasshoppers (Hegde, 1981; Mayya, 1995; Nishith \& Rai, 1999). Several industries like Manglaore Refinery and Petrochemicals Limited (MRPL), Mangalore Chemical and Fertilizers Limited (MCF) and other small industries discharge their effluents, polluting air and water. This is likely to have drastic effects on the grasshopper populations. Many bird species like parakeets, kingfishers, herons, mynas etc. are becoming rare in this region. To understand the impacts of such developmental activities on grasshoppers, which form an essential aspect of the food chain for birds, a preliminary survey was conducted between September 2000 and June 2002.

\section{Methodology}

The survey was conducted in places like Mangalore University Campus, Someshwara, Mangalore Refinery and Petroleum Limited area, Mangalore Chemicals and Fertilizers area, Ermal, Maravanthe, Bantwal, Sullia, Kudremukh, and St. Mary's Island covering a total distance of $250 \mathrm{~km}^{2}$ to know the distribution of these insect pests. Specimens were collected using hand nets, and were properly spread and mounted in insect storage boxes, labeled with scientific names, place and date of collection and deposited in the SVS College museum for future reference.

\section{Results And Discussion}

A total of 28 species of short-horned grasshoppers (Acrididae) collected from 12 localities are listed in Tables 1 and 3. The number and distribution of different species in different localities with their relative abundance and period of distribution during different months are shown in Tables 2 and 3.
From the present survey it appears that Acrida exaltata, Dociostaurus sp. and Stauroderus bicolor are relatively abundant than other species among subfamily Acridinae. Among the species from subfamily Oedipodinae, Morphacris fasciata, Dittopternis venusta, Oedaleus abruptus and Acrotylus humbertianus were very much abundant than the other species. As reflected in Table 2 with regard to the period of distribution, it has been found that Acridinae were abundant during January and February, whereas Oedipodinae were abundant during February and March while Catantopinae and Pyrgomorphinae were present in large numbers during December and January. Thus among different species, Spathosternum prassiniferum was the most abundant followed by Catantops pinguis innotabilis, both from subfamily Catantopinae. The fluctuations in climatic factors like humidity, temperature, light and edaphic factors could influence reproduction patterns, breeding behaviours and physiology of grasshoppers, which is reflected in the fluctuation in the relative abundance and species richness in terms of their occurrence and distribution. In the current study it appears that short horned grasshoppers are found to be most common during post monsoon season (Dec.-Feb.). Further, taxa belonging to the subfamily Catantopinae such as Catantops pinguis innotabilis, Spathosternum prassiniferum, Eyprepocnemis alacris alacris and Tylotropidius varicornis were found widely distributed while (Gelastorrhinus sempipictus and two species of Oxya in the same subfamily and Gastrimorgus africanus of the subfamily Oedipodinae were restricted in their distribution. It is also evident from the survey that more species diversity in Mangalagangotri Campus and Ermal region may be due to less anthropogenic activities. Further, it could be surmised that less species diversity in other localities are mainly due to pollution and agricultural practices.

\section{REFERENCES}

Hegde, M.J. (1981). Chromosomal studies in natural populations of some Acrididae and the effects of certain chemotherapeutic agents on chromosomes. $\mathrm{Ph} . \mathrm{D}$. Thesis submitted to Mangalore University (Unpublished).

Mayya, S. (1995). Cytogenetic studies of a few short-horned grasshoppers (Fam:Acrididae) occurring in Dakshina Kannada district of Karnataka State. M.Phil. Dissertation submitted to Mangalore University (Unpublished).

Nishitha and Yogitha Rai (1999). Studies on grasshopper species collected from Mangalore University Campus. M.Sc. Dissertation submitted to Mangalore University (Unpublished).

\section{ACKNOWLEDGEMENTS}

We thank the UGC for the financial assistance and Prof. Virakthamutt, G.K.V.K. Bangalore and Prof. T.C. Narendran, Calicut University, for their help in identifying the specimens.

(C) Zoo Outreach Organisation; www.zoosprint.org Manuscript 1068; Received 04 August 2003; Revised received 09 June 2005; Finally accepted 01 July 2005; Date of publication 21 August 2005 
Table 1. List of species of Acrididae collected between September 2000 and June 2002

\begin{tabular}{|c|c|c|c|c|c|}
\hline Species Name & Place of Collection & Months of & $\begin{array}{l}\text { Host } \\
\text { Collection }\end{array}$ & Size & Abundance \\
\hline \multicolumn{6}{|l|}{$\begin{array}{l}\text { Family: Acrididae } \\
\text { Subfamily: Acridinae }\end{array}$} \\
\hline Acrida turrita (Linn.) & M.C.F.Ermal & January, February & Green grass & Big $50 \mathrm{~mm}$ & Very common \\
\hline Acrida exaltata (Walk.) & M.C.F.Ermal & February, April & Green or dry grass & Big $53 \mathrm{~mm}$ & Very common \\
\hline Dociostaurus sp. & Mangala-gangothri & January, May, June & Dry grass or leaves & Average $23 \mathrm{~mm}$ & Common \\
\hline Dociostaurus apicalis (Walk.) & St. Mary's Island & February, March & Dry grass & Small 13mm & Common \\
\hline Stauroderus bicolor (Charp.) & $\begin{array}{l}\text { Mangala-gangothri, } \\
\text { Ermal, Kudremukh }\end{array}$ & March, November & Green or dry grass & Average $15-24 \mathrm{~mm}$ & Very common \\
\hline Aiolopus thalassinus tamulus (Fabr.) & Mangala-gangothri & January, March & Dry grass & Small $18 \mathrm{~mm}$ & Uncommon \\
\hline Aulacobothrus luteipes (Walk.) & Mangala-gangothri & February, December & Dry grass & Average $20 \mathrm{~mm}$ & Uncommon \\
\hline $\begin{array}{l}\text { Sub family Oedipodinae } \\
\text { Morphacris fasciata (Kirby) }\end{array}$ & Someshwara Gangothri & January, November & $\begin{array}{l}\text { Sandy shore } \\
\text { or Dry leaves }\end{array}$ & $\begin{array}{l}\text { Average or Big } \\
30 \mathrm{~mm} / 40 \mathrm{~mm}\end{array}$ & Very common \\
\hline Dittopternis zebrata (Walk.) & Mangala- gangothri, Ermal & February, March, April & Dry grass & Average $25 \mathrm{~mm}$ & Common \\
\hline Dittopternis venusta (Stal) & Ermal, Sullia & February, March & Rocky area (laterite) & Average $25-30 \mathrm{~mm}$ & Common \\
\hline Heteroptermis respondens (Sauss.) & MRPL, Swarga & March, April & Brown soil (rocky) & Average $19-22 \mathrm{~mm}$ & Uncommon \\
\hline Oedaleus abruptus (Thunb.) & Ermal, Mangala-gangothri & February, April & Dry grass, Rocky & Average $18-20 \mathrm{~mm}$ & Common \\
\hline Acrotylus humbertianus (Sauss.) & $\begin{array}{l}\text { Someshwara, Maravanthe, } \\
\text { Calicut }\end{array}$ & January, March & $\begin{array}{l}\text { Sandy beach with } \\
\text { black soil \& dry } \\
\text { vegetation }\end{array}$ & Average $18-20 \mathrm{~mm}$ & Common \\
\hline Gastrimargus africanus (Sauss.) & Kudremukh & January & Green or dry grass & Big $30-45 \mathrm{~mm}$ & Uncommon \\
\hline Leva indica (I.Bol.) & Mangala-gangothri & January, May, June & $\begin{array}{l}\text { Green grass or } \\
\text { dry leaves }\end{array}$ & Small 15mm & Common \\
\hline \multicolumn{5}{|l|}{ Catantopinae } & Common \\
\hline Oxya hyla hyla (Serv.) & Mangala-gangothri & December & Green grass & Average $25 \mathrm{~mm}$ & Common \\
\hline Oxya velox (Marschal) & $\begin{array}{l}\text { Mangala-gangothri, } \\
\text { Bantwal }\end{array}$ & November, December & $\begin{array}{l}\text { Green grass } \\
\text { or dry grass }\end{array}$ & $\begin{array}{l}\text { Slightly big } \\
16-32 \mathrm{~mm}\end{array}$ & Common \\
\hline Catantops pinguis innotabilis (Stal) & $\begin{array}{l}\text { Mangala-gangothri, } \\
\text { Ermal, Swarga }\end{array}$ & $\begin{array}{l}\text { February, March, } \\
\text { December }\end{array}$ & Dry leaves & $\begin{array}{l}\text { Slightly big or } \\
\text { big } 27-34 \mathrm{~mm}\end{array}$ & Very common \\
\hline $\begin{array}{l}\text { Xenocatantops humilis humilis } \\
\text { (Serv.) }\end{array}$ & Mangala-gangothri & $\begin{array}{l}\text { January, November, } \\
\text { December, }\end{array}$ & Hibiscus leaves & $\begin{array}{l}\text { Average (nymph } \\
\text { coloured) } 30 \mathrm{~mm}\end{array}$ & Uncommon \\
\hline Spathosternum prasiiferum (Walker) & $\begin{array}{l}\text { Mangala-gangothri, Ermal, } \\
\text { MRPL, Bantwal }\end{array}$ & $\begin{array}{l}\text { March, September, } \\
\text { October, November, }\end{array}$ & Green or dry grass & Small 13-20mm & Very common \\
\hline Gelastorrhinus semipictus (Walker) & Ermal & November & Tall green grass & Big 20-46mm & Uncommon \\
\hline Eyprepocnemis alacris alacris (Serv.) & $\begin{array}{l}\text { Ermal, MRPL, Swarga, } \\
\text { Mangala-gangothri }\end{array}$ & January, February & Dry grass & Big 35-38mm & Common \\
\hline Tylotropidius varicornis (Walker) & $\begin{array}{l}\text { Mangala-gangothri, Ermal, } \\
\text { Swarga }\end{array}$ & January & Dry grass & Quite big $28-48 \mathrm{~mm}$ & Common \\
\hline Epistaurus praemorsa (Stal) & Kudremukh & January & Dry grass & Average $15-18 \mathrm{~mm}$ & Uncommon \\
\hline \multicolumn{6}{|l|}{ Pyrgomorphinae } \\
\hline Chrotogonus oxypterus (Blanch) & Mangala-gangothri, Swarga & January, December & $\begin{array}{l}\text { Black/Red } \\
\text { rocky area }\end{array}$ & Small $13-19 \mathrm{~mm}$ & Common \\
\hline Attractomorpha crenulata (Fabr.) & $\begin{array}{l}\text { Bantwal, Mangala- } \\
\text { gangothri, MRPL }\end{array}$ & January, December & Green grass & Small $16-26 \mathrm{~mm}$ & Common \\
\hline $\begin{array}{l}\text { Neorthacris acuticeps acuticeps } \\
\text { (I.Bol.) }\end{array}$ & Mangala-gangothri & January, October & $\begin{array}{l}\text { Black soil with } \\
\text { green grass }\end{array}$ & Average $20-24 \mathrm{~mm}$ & Uncommon \\
\hline
\end{tabular}


Table 2. Relative abundance* of short-horned grasshopper species collected between September 2000 and June 2002.

\begin{tabular}{|c|c|c|c|c|c|c|c|c|c|c|}
\hline & Sep. & Oct & Nov & Dec & Jan & Feb & Mar & Apr & May & June \\
\hline \multicolumn{11}{|l|}{ Acridinae } \\
\hline Acrida turrita & - & - & - & - & + & + & - & - & - & - \\
\hline Acrida exaltata & - & - & - & - & + & +++ & + & + & - & - \\
\hline Dociostaurus sp. & - & - & - & - & +++ & + & + & - & - & - \\
\hline Dociostaurus apicalis & - & - & - & - & - & +++ & + & - & - & - \\
\hline Stauroderus bicolor & - & - & + & + & + & + & +++ & + & - & - \\
\hline Aiolopus thalassinus tamulus & - & - & - & - & + & + & + & - & - & - \\
\hline Aulacobothrus luteipes & - & - & - & $H$ & + & + & - & - & - & - \\
\hline \multicolumn{11}{|l|}{ Oedipodinae } \\
\hline Morphacris fasciata & - & - & + & +++ & $H$ & - & - & - & - & - \\
\hline Dittopternis venusta & - & - & - & - & + & +++ & + & +++ & - & - \\
\hline Dittopternis zebrata & - & - & - & - & - & +++ & +++ & + & - & - \\
\hline Heteropternis respondens & - & - & - & - & - & - & +t+ & + & - & - \\
\hline Oedalus abruptus & - & - & - & - & + & +++ & +++ & + & - & - \\
\hline Acrotylus humbertianus & - & - & - & + & ++++ & +++ & +++ & + & - & - \\
\hline Gastrimargus africanus & - & - & - & - & + & - & - & - & - & - \\
\hline Leva indica & - & - & - & - & +++ & + & - & - & + & + \\
\hline \multicolumn{11}{|l|}{ Catantopinae } \\
\hline Oxya fuscovittata & - & - & - & + & - & - & - & - & - & - \\
\hline Oxya hyla hyla & - & - & - & + & - & - & - & - & - & - \\
\hline Oxya velox & - & - & + & + & - & - & - & - & - & - \\
\hline Catantops pinguis innotabilis & - & - & - & H & + & ++++ & $H$ & - & - & - \\
\hline Xenocatantops humilis humilis & - & - & + & +++ & + & - & - & - & - & - \\
\hline Spathosternum prasiniferum & + & + & +++ & +++++ & + & + & - & - & - & - \\
\hline Gelastorrhinus semipictus & - & + & - & - & - & - & - & - & - & - \\
\hline Eyprepocnemis alacris alacris & - & - & - & - & + & +++ & + & - & - & - \\
\hline Tylotropidius varicornis & - & - & - & - & + & + & - & - & - & - \\
\hline Epistaurus praemorsa & - & - & - & - & + & + & - & - & - & - \\
\hline \multicolumn{11}{|l|}{ Pyrgomorphpinae } \\
\hline Chrotogonus oxypterus & - & - & - & + & +++ & + & - & - & - & - \\
\hline Attractomorpha crenulata & - & - & - & $H$ & + & - & - & - & - & - \\
\hline Neorthacris acuticeps acuticeps & - & + & - & + & H & - & - & - & - & - \\
\hline
\end{tabular}

* In one collection $+=<10 ;++=<30 ;+++=<50 ;++++=<70$

Table 3. Species of short-horned grasshoppers recorded from different localities.

\begin{tabular}{|c|c|c|c|c|c|c|c|c|c|c|c|c|}
\hline Species & $\begin{array}{l}\text { Mangala } \\
\text { gangothri }\end{array}$ & $\begin{array}{l}\text { Somes } \\
\text { hwara }\end{array}$ & MRPL & MCF & Ermal & $\begin{array}{l}\text { Place of } \\
\text { Bantwal }\end{array}$ & $\begin{array}{l}\text { Collectic } \\
\text { Swarga }\end{array}$ & $\begin{array}{l}\text { n } \\
\text { Marva } \\
\text { nthe }\end{array}$ & $\begin{array}{l}\text { Kudre } \\
\text { mukh }\end{array}$ & Sullia & Calicut & $\begin{array}{l}\text { St Mary } \\
\text { Island }\end{array}$ \\
\hline Acrida turrita & - & - & - & + & + & - & - & - & - & - & - & - \\
\hline Acrida exaltata & - & - & - & + & - & + & - & - & - & - & - & - \\
\hline Dociostaurus sp. & + & - & - & - & - & - & - & - & - & - & - & - \\
\hline Dociostaurus apicalis & - & - & - & - & - & - & - & - & - & - & - & + \\
\hline Stauroderus bicolor & + & - & - & - & + & - & - & - & + & - & - & - \\
\hline Aiolopus thalassinus tamulus & + & - & - & - & - & - & - & - & - & - & - & - \\
\hline Aulacobothrus luteipes & + & - & - & - & - & - & - & - & - & - & - & - \\
\hline Morphacris fasciata & + & + & + & - & - & - & - & - & - & - & - & - \\
\hline Dittopternis venusta & + & - & - & - & + & - & - & - & - & - & - & - \\
\hline Dittopternis zebrata & - & - & - & - & + & - & - & - & - & + & - & - \\
\hline Heteropternis respondens & - & - & + & - & - & - & + & - & - & - & - & - \\
\hline Oedalus abruptus & + & - & - & - & + & - & - & - & - & - & - & - \\
\hline Acrotylus humbertianus & - & + & - & - & - & - & - & + & - & - & + & - \\
\hline Gastrimargus africanus & - & - & - & - & - & - & - & - & + & - & - & - \\
\hline Leva indica & + & - & - & - & - & - & - & - & - & - & - & - \\
\hline Oxya fuscovittata & - & - & - & - & - & + & - & - & - & - & - & - \\
\hline Oxya hyla hyla & + & - & - & - & - & - & - & - & - & - & - & - \\
\hline Oxya velox & + & - & - & - & - & + & - & - & - & - & - & - \\
\hline Catantops pinguis innotabilis & + & - & - & - & + & - & + & - & - & - & - & + \\
\hline Xenocatantops humilis humilis & + & - & - & - & - & - & - & - & - & - & - & - \\
\hline Spathosternum prasiniferum & + & - & + & - & + & + & - & - & - & - & - & - \\
\hline Gelastorrhinus semipictus & - & - & - & - & + & - & - & - & - & - & - & - \\
\hline Eyprepocnemis alacris alacris & + & - & + & - & + & - & + & - & - & - & - & - \\
\hline Tylotropidius varicornis & + & - & - & - & + & - & + & - & - & - & - & - \\
\hline Epistaurus praemorsa & - & - & - & - & - & - & - & - & + & - & - & - \\
\hline Chrotogonus oxypterus & + & - & - & - & - & - & + & - & - & - & - & - \\
\hline Attractomorpha crenulata & + & - & + & - & - & + & - & - & - & - & - & - \\
\hline Neorthacris acuticeps acuticeps & + & - & - & - & - & - & + & - & - & - & - & - \\
\hline
\end{tabular}

